\title{
Patientensicherheit:
}

\section{Wo stehen wir heute?}

\section{Margrit Leutholda, Dieter Conen ${ }^{b}$, Kathrin Hirterc, Enea Martinellid}

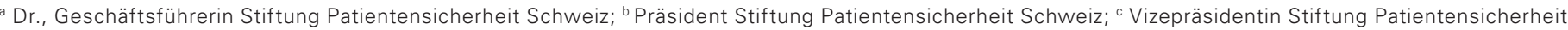
Schweiz; ${ }^{d}$ Dr., Vizepräsident Stiftung Patientensicherheit Schweiz

In den letzten Jahren hat sich auch in der Schweiz viel getan: Über Fehler darf gesprochen werden, fast alle Spitäler haben Fehlermeldesysteme. Dennoch gibt es grosse Aufgaben: Mit dem exponentiellen Wissenszuwachs, komplexeren Technologien und sozio-kulturellen Veränderungen hinken die erzielten Verbesserungen den immer neuen Herausforderungen hinterher. Die Stiftung Patientensicherheit Schweiz engagiert sich, um Betriebe und Fachpersonen zu unterstützen und befähigen.

Mit dem Erscheinen der Publikation To err is human des Amerikanischen Institute of Medicine im Jahr 2000 wurde weltweit ins Bewusstsein gerufen, dass auch in der Medizin häufig Fehler geschehen und Patienten durch die Gesundheitsversorgung Schaden erleiden.

\section{Um unerwünschte Ereignisse effektiv vermeiden zu können, ist eine prospektive Sicht notwendig.}

Internationale Untersuchungen zeigen, dass knapp $10 \%$ der hospitalisierten Patienten von einem unerwünschten Ereignis betroffen sind [1, 2]. Etwa die Hälfte davon gilt als vermeidbar. Etwa 0,1\% der hospitalisierten Patienten verstirbt an einem potentiell vermeidbaren unerwünschten Ereignis [2]. Seither hat ein langsamer, aber stetiger Kulturwandel eingesetzt. Die «systemische Fehlbarkeit der Gesundheitsversorgung» wird heute offen thematisiert; nicht nur bei den Gesundheitsfachpersonen, sondern auch durch die $\mathrm{Pa}$ tientinnen und Patienten und die Öffentlichkeit. Der Blick wird zunehmend vom Individuum weg hin auf die Organisation gelegt. Dadurch erfolgt auch eine Abkehr von der Schuldfrage. Es wird weniger die Frage nach dem "wer» gestellt, sondern vielmehr Fragen wie «Warum konnte dies geschehen?» oder "Wie ist es dazu gekommen?». Viele Spitäler haben Fehlermeldesysteme CIRS (Critical Incidence Reporting System) eingeführt, in die Meldungen von Fehlern oder Beinahe-Schädigungen anonym eingegeben werden können mit dem Ziel, solche Meldungen systematisch aufzuarbeiten, ins System zurückzuspiegeln und somit aus Fehlern zu lernen. Das Etablieren von
CIRS ist eine wichtige Grundlage; dessen Erfolg bzw. praktischer Nutzen hängt jedoch wesentlich davon $a b$, wie diese Systeme genutzt werden. Dabei sind insbesondere auch Parameter wie die Meldequalität und -frequenz entscheidend.

Zudem findet man heute auch in fast allen Spitälern ein klinisches Risikomanagement vor mit dem expliziten Auftrag, die Patientensicherheit zu erhöhen. All diese Bemühungen zielen in die richtige Richtung und sind wichtige Bausteine zum Erreichen einer verbesserten Sicherheitskultur.

Die Möglichkeit, in der eigenen Organisation Fehler oder Beinahe-Fehler zu thematisieren, hängt sehr stark von der vorherrschenden Kultur ab. Eine entscheidende Rolle kommt dabei den Führungspersonen zu. Wenn das Betriebsklima zulässt, dass Fehler thematisiert werden dürfen, Speak-Up ermuntert wird (siehe dazu auch den Artikel von D. Schwappach et al. in dieser Ausgabe), Gefässe wie z.B. Mortalitäts- und Morbiditätskonferenzen etabliert und richtig genutzt werden, und nicht nur den Sorgen und Nöten der von einem Zwischenfall betroffenen Patientinnen und Patienten, sondern auch

\section{Die im Jahr 2003 gegründete Stiftung ist} das schweizweite Kompetenzzentrum für alle Belange der Patientensicherheit.

den Mitarbeitenden als "second victims» Rechnung getragen wird, dann sind entscheidende Schritte hin zur Sicherheitskultur getan. Wie wir wissen, ist dies jedoch noch längst nicht in allen Betrieben der Fall. Hemmende Faktoren können ein fehlendes Problembewusstsein bei Führungspersonen oder strenge Hier- 


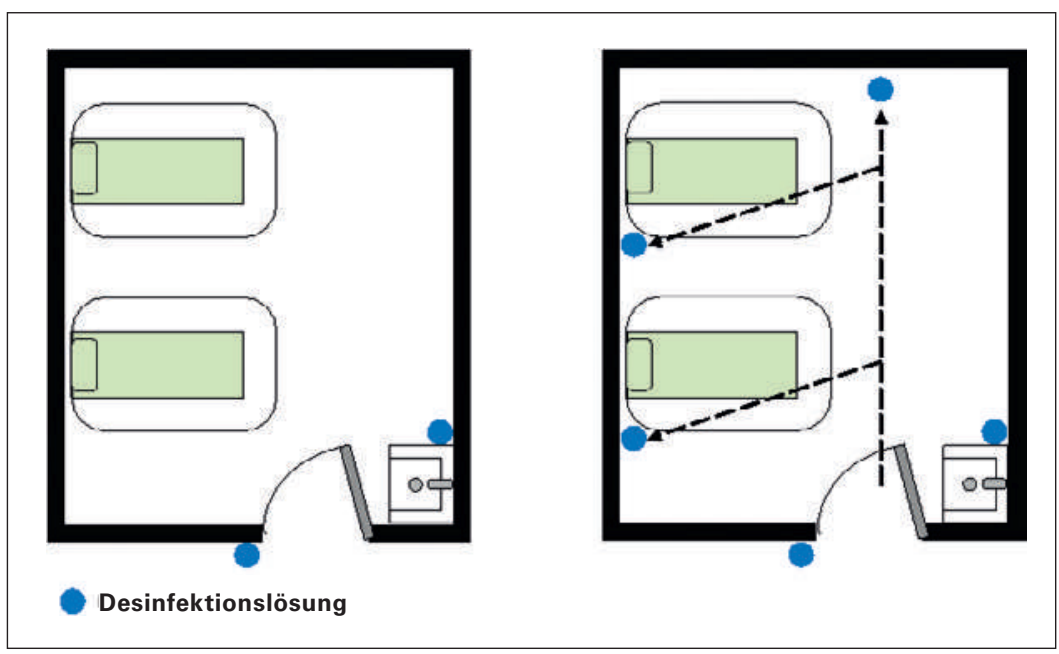

Einfache Massnahme zur Steigerung der Handhygiene: Ist der Spender im Blickfeld (rechts), steigt die Compliance von 12 auf 56\%.

archien, aber auch ein steigender ökonomischer Druck sein, der weder Zeit noch Raum lässt, um eine nachhaltige Sicherheitskultur zu entwickeln.

Analysen von Fehlern oder Beinahe-Fehlern sind meist retrospektiv. Um unerwünschte Ereignisse effektiv vermeiden zu können, ist eine prospektive Sicht notwendig, um mögliche Schwachstellen im System, die eine Fehleranfälligkeit begünstigen, zu orten und zu eliminieren

Ein verbreiteter Ansatz, um die Patientensicherheit zu verbessern, sind einzelne Interventionen oder Interventionsbündel, die für spezifische Problemfelder entwickelt und eingesetzt werden. Internationale Studien zeigen, dass mit solchen Massnahmen zumindest kurzfristig überzeugende Erfolge erzielt werden können. Dank der interdisziplinären Anlage, sowie spezifischen Trainings und Schulungen kann auch die TeamZusammenarbeit und die Kommunikation verbessert werden, beides entscheidende Komponenten für die Reduktion vermeidbarer unerwünschter Ereignisse.

\section{Dreizehn Prozent aller Schweizerinnen und Schweizer geben an, dass bei ihnen kürzlich ein Behandlungsfehler aufgetreten ist.}

Da diese Art von Verbesserungsprogrammen wesentlich auch auf die Verhaltensänderungen der Mitarbeitenden bauen, ist der nachhaltige, andauernde Erfolg jedoch noch ungewiss. Aus der Psychologie und den eigenen alltäglichen Erfahrungen wissen wir, dass es extrem schwierig ist, Verhalten zu ändern. Ein Monitoring nach Programmabschluss erscheint zwingend, um verfolgen zu können, ob erzielte Veränderungen und Verbesserungen denn auch tatsächlich nachhaltig etabliert werden konnten.
Ein weiterer, vielversprechender Ansatz zielt darauf hin, das betriebliche Umfeld so zu gestalten, dass es einfacher wird, sich «richtig» zu verhalten. Klar geregelte innerbetriebliche Abläufe und Prozesse, die Anordnung und Erreichbarkeit von Materialien, eine Umgebungsgestaltung, in der Faktoren wie Licht, Lärm usw. optimiert werden, können ebenfalls entscheidend beitragen, dass weniger Fehler entstehen. So konnte z.B. gezeigt werden, dass die HändehygieneCompliance von 12 auf 56\% gesteigert werden kann, wenn die Dispenser mit der Desinfektionslösung so angebracht sind, dass sie den behandelnden Personen unmittelbar ins Auge springen [1] (s. Abb.). Ist der Spender in den entscheidenden Momenten im Blickfeld, wird er vom Personal automatisch häufiger genutzt, ohne dass es hierfür eine bewusste kognitive Anstrengung benötigt. Zur Erreichung einer besseren Patientensicherheit muss zukünftig das Potential solcher «Verhältnisprävention» stärker genutzt werden.

\section{Der Beitrag von Patientensicherheit Schweiz}

Die im Jahr 2003 gegründete Stiftung ist das schweizweite Kompetenzzentrum für alle Belange der Patientensicherheit. Sie will in erster Linie die Leistungserbringer in ihrem Engagement für eine verbesserte Sicherheitskultur unterstützen und befähigen. Sie generiert anhand von Studien Wissen und leitet daraus neue Erkenntnisse und praktische Handlungsempfehlungen ab, die durch Kurse, Tagungen und Schulungen verbreitet werden. Zudem führt sie seit $2012 \mathrm{im} \mathrm{Rah-}$ men der Qualitätsstrategie des Bundes in verschiedenen Hotspots der Patientensicherheit Pilotprogramme «progress!» mit ausgewählten Betrieben durch. Das erste "progress!»-Programm Sichere Chirurgie findet diesen Sommer seinen Abschluss, das zweite Programm Sichere Medikation an Schnittstellen wurde 2014 initiiert. Zudem sind zwei neue Programme zu folgenden Themen im Aufbau: Sicherheit bei Blasenkathetern (siehe dazu auch Artikel S. 1358 in dieser Ausgabe) und Sichere Medikation in Pflegeheimen.

Die Kernaktivitäten der Stiftung beruhten in der Vergangenheit primär auf Problemen in der stationären Akutversorgung. Die 2011 publizierte und massgeblich durch die FMH finanzierte Studie von Gehring und Schwappach nahm sich erstmals den Hotspots und der Sicherheit in der Grundversorgung an [2]. Auch die Rolle der Offizinapotheken für eine sichere Arzneimittelversorgung speziell der Migrationsbevölkerung wurde in den letzten Jahren untersucht und wichtige Erkenntnisse über spezielle Risiken wurden gewonnen [3]. Mit der Verlagerung von auch grossen invasiven 
Eingriffen und anderen vormals traditionell stationär durchgeführten Behandlungen gewinnt der ambulante Bereich zunehmend an Bedeutung und es stellen sich neue Sicherheitsfragen. Auch da wird die Stiftung künftig aktiv werden.

In diesem Jahr machen wir zudem erstmals eine Auslegeordnung zu Patientensicherheitsaspekten in der Psychiatrie und Langzeitpflege. Gemeinsam mit Expertinnen und Experten aus verschiedensten Fachdisziplinen erörtern wir die Besonderheiten in den jeweiligen Bereichen und werden daraus prioritäre Handlungsfelder und entsprechende Projekte ableiten.

Die von Patientensicherheit Schweiz angebotenen Kurse "Täter als Opfer», "Error and Risk Analysis» sowie «Kommunikation mit Patienten und Angehörigen nach einem Zwischenfall» erfreuen sich nach wie vor einer regen Nachfrage. Neu entwickelt wird zudem ein Modul für Führungsgremien von Spitälern.

Mit der erstmals von Patientensicherheit Schweiz initiierten und durchgeführten Aktionswoche Patientensicherheit 2015 stellt sie den Leistungserbringern eine Plattform zur Verfügung, um bereits Geleistetes darzustellen.

Sie will in Kooperation mit zahlreichen Akteuren ein Zeichen für eine verbesserte Patientensicherheit setzen und die Öffentlichkeit informieren. Mannigfache Aktionen sollen aufzeigen, welche Themen zentral sind, was zur Förderung der Patientensicherheit durch die Leistungserbringer getan wird, wo dringender Handlungsbedarf besteht und wo weitere Anstrengungen unternommen werden müssen.

Ziel dieser Aktionswoche ist es, das Thema Patientensicherheit sowohl in allen Gesundheitseinrichtungen wie auch in der Öffentlichkeit noch breiter zu lancieren und auf die gesundheitspolitische Bedeutung hinzuweisen.

Die Aktionswoche ist eine wichtige Gelegenheit, Patientinnen und Patienten und Angehörige über sicherheitsrelevante Aspekte zu informieren. Dass dies ein Thema für die Bürgerinnen und Bürger ist, zeigt eine vergleichende Länderstudie: Dreizehn Prozent aller Schweizerinnen und Schweizer geben an, dass bei ihnen kürzlich ein Behandlungsfehler aufgetreten ist, in England ist diese Quote mit 7,8\% am tiefsten, in Norwegen mit 21,1\% am höchsten [4].

\section{Ein Ausblick}

Patientensicherheit ist und bleibt eines der wesentlichen Themen in der modernen Medizin.

Mit dem exponentiellen Wissenszuwachs, den zunehmend komplexeren Technologien und Abläufen sowie den sozio-kulturellen Veränderungen in der Medizin hinken die erzielten Verbesserungen den immer neuen Herausforderungen hinterher.

Die Stiftung Patientensicherheit Schweiz wird sich denn auch in Zukunft den bestehenden und neuen Herausforderungen auf der ganzen Breite der medizinischen Behandlungskette annehmen und im Rahmen ihrer Möglichkeiten einen Beitrag leisten, damit die Medizin nicht nur besser (und teurer), sondern auch sicherer wird.

Mit dem im letzten Jahr lancierten Gesetzesentwurf als Grundlage für ein «Nationales Zentrum für Qualität und Patientensicherheit» ist die Thematik auch auf der politischen Agenda angelangt. Wir hoffen, dass künftig die Stiftung eine der Brisanz des Themas angemessene Grundfinanzierung erhält, um mit noch mehr Schlagkraft ihren wichtigen Beitrag leisten zu können.

Der FMH danken wir, dass sie im Rahmen unserer ersten Aktionswoche dieses Heft ganz der Patientensicherheit widmet.

\section{Literatur}

1 Birnbach DJ, Nevo I, Scheinman SR, Fitzpatrick M, Shekhter I, Lombard JL. Patient safety begins with proper planning: a quantitative method to improve hospital design. Qual Saf Health Care. 2010;19(5):462-5.

2 Gehring K, Schwappach D, Battaglia M, Buff R, Huber F, Sauter P, et al. Frequency of and Harm Associated With Primary Care Safety Incidents. Am J Manag Care. 2012;18(9):e323-e337.

3 Schwappach D, Meyer Massetti C, Gehring K. Communication barriers in counselling foreign-language patients in public pharmacies: threats to patient safety? International Journal of Clinical Pharmacy. 2012;34(5):765-72.

4 Schwappach DLB. Risk factors for patient-reported medical errors in eleven countries. Health Expect. 2014;17(3):321-31.

\section{Bildnachweis:}

D. Schwappach 